\title{
A WEAK-TYPE ESTIMATE FOR FOURIER-BESSEL MULTIPLIERS
}

\author{
JOHN GOSSELIN AND KRZYSZTOF STEMPAK
}

(Communicated by Richard R. Goldberg)

\begin{abstract}
We apply Hörmander's technique to prove a weak-type $(1,1)$ estimate for multiplier operators with respect to the Fourier-Bessel transform. This improves a result in $[4,5]$.
\end{abstract}

\section{INTRODUCTION}

Consider the measure space $\left(\mathbf{R}^{+}, d \mu(x)\right)$ where $d \mu(x)=x^{r} d x, \quad r$ being a fixed positive real number. By $L^{p}(\mu), 1 \leq p \leq \infty$, we denote the corresponding Lebesgue spaces equipped with the norms

$$
\|f\|_{p}=\left(\int_{0}^{\infty}|f|^{p} d \mu\right)^{1 / p}
$$

Let $\hat{f}(\lambda), \lambda \geq 0$, denote the Fourier-Bessel transform of the function $f \in$ $L^{1}(\mu)$. Explicitly this means

$$
\hat{f}(\lambda)=\int_{0}^{\infty} f(x) \phi_{\lambda}(x) d \mu(x)
$$

where $\phi_{\lambda}(x)=a(r)(\lambda x)^{-(r-1) / 2} J_{(r-1) / 2}(\lambda x), \quad x \geq 0, a(r)=2^{(r-1) / 2} \Gamma((r+1) / 2)$, and $J_{\alpha}$ denotes the Bessel function of the first kind of order $\alpha$. The functions $\phi_{\lambda}, \lambda \geq 0$, are eigenfunctions of the second order Bessel differential operator

$$
L=-\left(\frac{d}{d x^{2}}+\frac{r}{x} \frac{d}{d x}\right) \text {. }
$$

More precisely, we have

$$
L \phi_{\lambda}=\lambda^{2} \phi_{\lambda}
$$

If $r=n-1, n \geq 2$ being an integer, then

$$
\hat{f}(\|\bar{\lambda}\|)=\frac{1}{2} \pi^{-n / 2} \Gamma\left(\frac{n}{2}\right) \int_{\mathbf{R}^{n}} f(\|\bar{x}\|) \exp (-i\langle\bar{\lambda}, \bar{x}\rangle) d \bar{x}
$$

Received by the editors May 22, 1987.

1980 Mathematics Subject Classification (1985 Revision). Primary 42B15; Secondary 42C15.

Key words and phrases. Fourier-Bessel transform, multipliers, generalized convolution. 
for $\bar{\lambda} \in \mathbf{R}^{n}$. Thus $\hat{f}$ gives the radial part of the Fourier transform of the radial function $f(\|\bar{x}\|)$ on $\mathbf{R}^{n}$ (observe that $f \in L^{1}(\mu)$ if and only if $f(\|\bar{x}\|) \in$ $L^{1}\left(\mathbf{R}^{n}, d \bar{x}\right)$ where $\|\cdot\|$ denotes the Euclidean norm and $d \bar{x}$ is Lebesgue measure on $\mathbf{R}^{n}$ ). In the case $r=n-1$, one can easily see that for the Fourier-Bessel transform, both the inversion theorem and Plancherel's formula hold. In particular we have

$$
f(x)=a(r)^{-1} \int_{0}^{\infty} \hat{f}(\lambda) \phi_{\lambda}(x) d \mu(\lambda)
$$

almost everywhere on $\mathbf{R}^{+}$providing $f \in L^{1}(\mu)$ and $\hat{f} \in L^{1}(\mu)$. For $f \in L^{2}(\mu)$ we have

$$
\int_{0}^{\infty}|f(x)|^{2} d \mu(x)=a(r)^{-2} \int_{0}^{\infty}|\hat{f}(\lambda)|^{2} d \mu(\lambda) .
$$

The above results for general $r>0$ can be established via a summability argument similar to that in the case of the ordinary Fourier transform. The key idea is that the Gaussian is invariant under the Fourier-Bessel transform when appropriately normalized. We also note that in the case $r=n-1$, the operator $-L$ is the radial part of the ordinary Laplace operator on $\mathbf{R}^{n}$. Finally, we mention that $C$ will be used to denote a constant which may vary from line to line.

For any bounded function $m$ on $(0, \infty)$ we define the multiplier operator $T_{m}$ by $\left(T_{m} f\right)^{\wedge}=m \hat{f}$. The aim of this note is to prove the following theorem which is a generalization of the celebrated Hörmander-Mihlin multiplier theorem for the Fourier transform (see [3]).

Theorem 1.1. Let $k$ denote the least even integer $>(r+1) / 2$ and let $m \in$ $C^{k}(0, \infty)$ be a bounded function which satisfies

$$
\left.\left(\int_{R / 2}^{R}\left|m^{(s)}(\lambda)\right|^{2} d \mu(\lambda)\right)\right)^{1 / 2} \leq B R^{(r+1) / 2-s},
$$

where $B$ is a constant independent of $R>0$ and $s=0,1, \ldots, k$. Then the operator $T_{m}$ is of weak-type $(1,1)$, i.e.

$$
\mu\left(\left\{x \in(0, \infty):\left|T_{m} f(x)\right|>\alpha\right\}\right) \leq C \alpha^{-1}\|f\|_{1}
$$

where $C$ is independent of $\alpha>0$ and $f \in L^{1}(\mu)$.

Using the interpolation theorem of Marcinkiewicz and duality we also obtain the following

Corollary 1.2. Let $m$ satisfy the assumptions of the Theorem 1.1. Then for $1<p<\infty, T_{m}$ is of strong type $(p, p)$, i.e. $\left\|T_{m} f\right\|_{p} \leq C_{p}\|f\|_{p}$.

Remark. The result of the above corollary was obtained previously by the second author by means of a Littlewood-Paley theory for the Fourier-Bessel transform $($ see $[4,5])$. The proof of the above theorem is more direct and is based upon a generalized convolution structure which is natural for the Fourier-Bessel 
transform. The novel ingredient of our proof is a Bernstein-type inequality for this convolution structure. We establish this inequality in the following section.

\section{A BERNSTEIN-TYPE INEQUALITY FOR GENERALIZED TRANSLATIONS}

Since Hirschman and Bochner considered the Banach algebra of radial functions over thirty years ago, it has been known that $L^{1}(\mu)$ admits a commutative Banach algebra structure for which the Fourier-Bessel transform becomes the Gelfand transform (see [4 and 5] for details and for other references). In order to describe this structure we define the generalized translation operator $T^{y}$, $y \geq 0$, for suitable functions $f$, by

$$
T^{y} f(x)=\int_{0}^{\pi} f\left((x, y)_{\theta}\right) d \nu(\theta)
$$

where $(x, y)_{\theta}=\left(x^{2}+y^{2}-2 x y \cos \theta\right)^{1 / 2}, \quad 0 \leq \theta \leq \pi, x, y \geq 0$ and $d \nu(\theta)$ denotes the probability measure $b(r)(\sin \theta)^{r-1} d \theta$ on $[0, \pi]$. Here

$$
b(r)=\pi^{-1 / 2} \Gamma((r+1) / 2) \Gamma(r / 2)^{-1} .
$$

The operator $T^{y}$ can also be described by

$$
T^{y} f(x)=\int_{|x-y|}^{x+y} f(z) d W_{x, y}(z)
$$

where the probability measure $d W_{x, y}(z)$ is supported on $[|x-y|, x+y]$ and given by

$$
d W_{x, y}(z)=c(r) \frac{\Delta(x, y, z)^{r-2}}{(x y z)^{r-1}} d \mu(z),
$$

where $c(r)=2^{r-2} \Gamma((r+1) / 2) \Gamma(r / 2)^{-1} \pi^{-1 / 2}$. In the above formula $x, y, z \geq$ $0,|x-y| \leq z \leq x+y$, and $\Delta(x, y, z)$ denotes the area of a triangle with sides $x, y, z$. It is quite straightforward to go from (2.2) to (2.1) by a change of variables. Next, it is easy to check that the measure $d W_{x, y}(z) d \mu(y)$ is symmetric with respect to $y$ and $z$, i.e. $d W_{x, y}(z) d \mu(y)=d W_{x, z}(y) d \mu(z)$. From this it follows that the operators $T^{y}, y \geq 0$, are selfadjoint on $L^{2}(\mu)$ and moreover that

$$
\int_{0}^{\infty} f(x) T^{y} g(x) d \mu(x)=\int_{0}^{\infty} T^{y} f(x) g(x) d \mu(x),
$$

for any reasonable pair of functions $f$ and $g$, e.g. if $f \in L^{\infty}(\mu)$ and $g \in$ $L^{1}(\mu)$. It is also easy to see that generalized translations are submarkovian contractions, i.e. $0 \leq f \leq 1$ implies $0 \leq T^{y} f \leq 1$ and $\left\|T^{y} f\right\|_{p} \leq\|f\|_{p}$, for $1 \leq p \leq \infty$. We now define the generalized convolution $f * g$ for $f, g \in L^{1}(\mu)$ by

$$
f * g(x)=\int_{0}^{\infty} f(y) T^{y} g(x) d \mu(y) .
$$


It is well known that the following identity involving the functions $\phi_{\lambda}$ and the generalized translations $T^{y}$ is valid

$$
T^{y} \phi_{\lambda}(x)=\phi_{\lambda}(x) \phi_{\lambda}(y)
$$

(see $[4,5]$ for references). Using $(2.3)$ and $(2.5)$ one can easily verify that for any $f, g \in L^{1}(\mu)$ we have $(f * g)^{\wedge}=\hat{f} \hat{g}$ and $\left(T^{y} f\right)^{\wedge}(\lambda)=\phi_{\lambda}(y) \hat{f}(\lambda)$. Therefore

$$
T^{y} g * f=g * T^{y} f \text {. }
$$

It is also easy to check that with the pseudodistance $\rho(x, y)=|x-y|$, the space $\left(\mathbf{R}^{+}, \mu, \rho\right)$ becomes a space of homogeneous type in the sense of [1]. Finally, from (2.2) it is immediate that $T^{y} f(x)=0$ if $f$ vanishes on the interval $(|x-y|, x+y)$ and that $\left|T^{y} f(x)\right| \leq T^{y}(|f|)(x)$ for $x, y \geq 0$.

In the proof of the Hörmander-Mihlin theorem (cf. [3]), Bernstein's inequality plays an important role. We now prove a Bernstein-type inequality for the generalized translations $T^{y}, y \geq 0$ :

Theorem 2.1. Suppose $h \in L^{1}(\mu)$ is a differentiable function on $\mathbf{R}^{+}$with $h^{\prime}(x) \in$ $L^{1}(\mu)$. Then

$$
\left\|T^{y_{1}} h-T^{y_{2}} h\right\|_{1} \leq\left\|h^{\prime}\right\|_{1}\left|y_{1}-y_{2}\right| .
$$

Proof. Using (2.1) we write

$$
\begin{aligned}
\left\|T^{y_{1}} h-T^{y_{2}} h\right\|_{1} & =\int_{0}^{\infty}\left|\int_{0}^{\pi}\left[h\left(\left(x, y_{1}\right)_{\theta}\right)-h\left(\left(x, y_{2}\right)_{\theta}\right)\right] d \nu(\theta)\right| d \mu(x) \\
& \leq \int_{0}^{\infty} \int_{0}^{\pi}\left|h\left(\left(x, y_{1}\right)_{\theta}\right)-h\left(\left(x, y_{2}\right)_{\theta}\right)\right| d \nu(\theta) d \mu(x) \\
& =\int_{0}^{\infty} \int_{0}^{\pi}\left|\int_{0}^{1} \frac{d}{d s}\left(h\left(\left(x, y_{2}+s\left(y_{1}-y_{2}\right)\right)_{\theta}\right)\right) d s\right| d \nu(\theta) d \mu(x) .
\end{aligned}
$$

For fixed $x, y_{1}, y_{2} \geq 0$ and $\theta \in(0, \pi]$, let

$$
\Phi(s) \equiv \Phi_{x, \theta}^{y_{1}, y_{2}}(s)=\left(x, y_{2}+s\left(y_{1}-y_{2}\right)\right)_{\theta}, \quad s \in[0,1] .
$$

Then

$$
\left|\frac{d}{d s} \Phi(s)\right| \leq\left|y_{1}-y_{2}\right|
$$

independently of $x, y_{1}, y_{2}, \theta$. To verify (2.9) consider the vectors $X=(x, 0)$, $Y_{i}=\left(y_{i} \cos \theta, y_{i} \sin \theta\right), \quad i=1,2$, in $\mathbf{R}^{2}$. Letting $\|\cdot\|$ denote the Euclidean norm on $\mathbf{R}^{2}$ it is easy to see that

$$
\left(x, y_{2}+s\left(y_{1}-y_{2}\right)\right)_{\theta}=\left\|Y_{2}+s\left(Y_{1}-Y_{2}\right)-X\right\| \equiv\left\|\xi_{s}\right\| .
$$

Since $\nabla(\|\cdot\|)(\xi)=\xi /\|\xi\|$ for $\xi \in \mathbf{R}^{2}$, Schwarz' inequality implies

$$
\begin{aligned}
\left|\frac{d}{d s} \boldsymbol{\Phi}(s)\right| & =\left|\frac{d}{d s}\left(\left\|Y_{2}+s\left(Y_{1}-Y_{2}\right)-X\right\|\right)\right|=\left|\left\langle\frac{\xi_{s}}{\left\|\xi_{s}\right\|}, Y_{1}-Y_{2}\right\rangle\right| \\
& \leq\left\|Y_{1}-Y_{2}\right\|=\left|y_{1}-y_{2}\right| .
\end{aligned}
$$


Here $\langle\cdot, \cdot\rangle$ denotes the ordinary Euclidean inner product on $\mathbf{R}^{2}$. This gives (2.9). Now, using (2.9) and the contraction property of generalized translations, we have from $(2.8)$

$$
\begin{aligned}
\| T^{y_{1}} h & -T^{y_{2}} h \|_{1} \\
& \leq\left|y_{1}-y_{2}\right| \int_{0}^{1} \int_{0}^{\infty} \int_{0}^{\pi}\left|h^{\prime}\left(\left(x, y_{2}+s\left(y_{1}-y_{2}\right)\right)_{\theta}\right)\right| d \nu(\theta) d \mu(x) d s \\
& \leq\left|y_{1}-y_{2}\right| \int_{0}^{1}\left\|T^{y_{1}+s\left(y_{1}-y_{2}\right)}\left(\left|h^{\prime}\right|\right)\right\|_{1} d s \\
& \leq\left|y_{1}-y_{2}\right|\left\|h^{\prime}\right\|_{1} .
\end{aligned}
$$

This completes the proof of Theorem 2.1.

As in the case of the Fourier transform (cf. [2]) Theorem 2.1 implies the following corollary. We include its proof for the sake of completeness.

Corollary 2.2. There exists a constant $C>0$ such that for any $\lambda>0$ and any function $f \in L^{1}(\mu)$ with $\operatorname{supp} \hat{f} \subset(0, \lambda)$

$$
\left\|T^{y_{1}} f-T^{y_{2}} f\right\|_{1} \leq C \lambda\left|y_{1}-y_{2}\right|\|f\|_{1} .
$$

Proof. Choose $\chi \in C^{\infty}\left(\mathbf{R}^{+}\right)$such that $\chi(\lambda)=1$ for $0<\lambda \leq 1$ and $\chi(\lambda)=0$ for $\lambda \geq 2$. Then by the inversion theorem we have $\chi=\hat{h}$ for some $C^{\infty}$ function $h \in L^{1}(\mu)$ with $h^{\prime} \in L^{1}(\mu)$. Let $h_{\varepsilon}(x)=\varepsilon h(\varepsilon x)$ for $\varepsilon>0$. Then $\hat{h}_{\varepsilon}(\lambda)=\chi(\lambda / \varepsilon)=1$ for $0 \leq \lambda \leq \varepsilon$. Thus, for $\varepsilon=\lambda$ we have

$$
T^{y_{1}} f-T^{y_{2}} f=h_{\lambda} *\left(T^{y_{1}} f-T^{y_{2}} f\right)=\left(T^{y_{1}} h_{\lambda}-T^{y_{2}} h_{\lambda}\right) * f
$$

and therefore by (2.7) we have

$$
\begin{aligned}
\left\|T^{y_{1}} f-T^{y_{2}} f\right\|_{1} & \leq\left\|T^{y_{1}} h_{\lambda}-T^{y_{2}} h_{\lambda}\right\|_{1}\|f\|_{1}=\left\|T^{\lambda y_{1}} h-T^{\lambda y_{2}} h\right\|_{1}\|f\|_{1} \\
& \leq \lambda \mid y_{1}-y_{2}\left\|h^{\prime}\right\|_{1}\|f\|_{1} .
\end{aligned}
$$

This establishes (2.10) with $C=\left\|h^{\prime}\right\|_{1}$ and completes the proof of the corollary.

\section{Proof of Theorem 1.1}

We closely follow the original proof of Hörmander (see [3, pp. 121-123]). Choose a function $\psi \in C_{0}^{\infty}\left(\mathbf{R}^{+}\right)$with support in $\left(\frac{1}{2}, 2\right)$ such that $\sum_{-\infty}^{\infty} \psi\left(2^{-j} \lambda\right)=1, \lambda>0$, and let

$$
m_{j}(\lambda)=m(\lambda) \psi\left(2^{-j} \lambda\right) \equiv m(\lambda) \psi_{j}(\lambda)
$$

and

$$
k_{j}(x)=\check{m}_{j}(x)=a(r)^{-1} \int_{0}^{\infty} m_{j}(\lambda) \phi_{\lambda}(x) \lambda^{r} d \lambda .
$$

Then $T_{m}=\sum_{-\infty}^{\infty} T_{m_{j}}$ where $T_{m_{j}} f=k_{j} * f$ and $k_{j} \in L^{1}(\mu)$. In order to prove the weak-type $(1,1)$ inequality $(1.3)$, it suffices to establish (see [1, p. 75])

$$
\sum_{j=-\infty}^{\infty} \int_{\left|x-y_{0}\right|>2\left|y-y_{0}\right|}\left|T^{y} k_{j}(x)-T^{y_{0}} k_{j}(x)\right| d \mu(x) \leq C,
$$


where $C$ is independent of $y, y_{0} \geq 0$. This will be implied in a standard way by the following estimates:

$$
\int_{\left|x-y_{0}\right| \geq 2\left|y-y_{0}\right|}\left|T^{y} k_{j}(x)-T^{y_{0}} k_{j}(x)\right| d \mu(x) \leq C\left(2^{j}\left|y-y_{0}\right|\right)^{(r+1) / 2-k},
$$

which is good when $2^{j}\left|y-y_{0}\right| \geq 1$, and

$$
\int_{\left|x-y_{0}\right| \geq 2\left|y-y_{0}\right|}\left|T^{y} k_{j}(x)-T^{y_{0}} k_{j}(x)\right| d \mu(x) \leq C 2^{j}\left|y-y_{0}\right|,
$$

which is good when $2^{j}\left|y-y_{0}\right|<1$.

To establish (3.4) we first note that conditions (1.2) imply

$$
\left(\int_{0}^{\infty}\left|L^{l} m_{j}(\lambda)\right|^{2} d \mu(\lambda)\right)^{1 / 2} \leq C B\left(2^{j}\right)^{(r+1) / 2-2 l}, \quad l=0,1, \ldots, k / 2 .
$$

In fact, by induction, one can easily verify the following Leibniz rule for $L$ : for $f, g \in C^{2 l}\left(\mathbf{R}^{+}\right)$

$$
L^{l}(f g)=\sum_{0<\alpha+\beta \leq 2 l} C_{\alpha, \beta}^{l} f^{(\alpha)} g^{(\beta)} \lambda^{\alpha+\beta-2 l} .
$$

Thus, taking $R=2^{j}$ in (1.2), we can estimate the left-hand side of (3.6) by

$$
\begin{aligned}
C & \sum_{0<\alpha+\beta \leq 2 l}\left(\int_{0}^{\infty}\left|m^{(\alpha)}(\lambda) \psi_{j}^{(\beta)}(\lambda) \lambda^{\alpha+\beta-2 l}\right|^{2} d \mu(\lambda)\right)^{1 / 2} \\
& \leq C \sum_{0<\alpha+\beta \leq 2 l} 2^{-\beta j}\left(\int_{2^{j-1}}^{2^{j+1}}\left|m^{(\alpha)}(\lambda) \lambda^{\alpha+\beta-2 l}\right|^{2} d \mu(\lambda)\right)^{1 / 2} \\
& \leq C \sum_{0<\alpha+\beta \leq 2 l}\left(2^{j}\right)^{\alpha-2 l}\left(\int_{2^{j-1}}^{2^{j+1}}\left|m^{(\alpha)}(\lambda)\right|^{2} d \mu(\lambda)\right)^{1 / 2} \\
& \leq C B\left(2^{j}\right)^{(r+1) / 2-2 l} .
\end{aligned}
$$

This gives (3.6). Next, using the Plancherel theorem and (3.6) we obtain

$$
\begin{aligned}
\|(1 & \left.+\left(2^{j} x\right)^{2}\right)^{k / 2} k_{j}\left\|_{2}=\right\|\left[\left(1+2^{2 j} L\right)^{k / 2} m_{j}\right]^{\vee} \|_{2} \\
& =a(r)^{-2}\left\|\left(1+2^{2 j} L\right)^{k / 2} m_{j}\right\|_{2} \\
& \leq C \sum_{l=0}^{k / 2}\left(2^{2 j}\right)^{l}\left\|L^{l} m_{j}\right\|_{2} \leq C B\left(2^{j}\right)^{(r+1) / 2} .
\end{aligned}
$$

Thus by Schwarz' inequality and the estimate $\left\|\left(1+\left(2^{j} x\right)^{2}\right)^{-k / 2}\right\|_{2} \leq$ $C\left(2^{j}\right)^{-(r+1) / 2}$ we obtain

$$
\begin{aligned}
\left\|k_{j}\right\|_{1} & \leq\left\|\left(1+\left(2^{j} x\right)^{2}\right)^{k / 2} k_{j}\right\|_{2}\left\|\left(1+\left(2^{j} x\right)^{2}\right)^{-k / 2}\right\|_{2} \\
& \leq C B .
\end{aligned}
$$


Moreover, since $\left\|\left(2^{j} x\right)^{k} k_{j}\right\|_{2} \leq\left\|\left(1+\left(2^{j} x\right)^{2}\right)^{k / 2} k_{j}\right\|_{2}$ we obtain in the same way

$$
\begin{aligned}
\int_{x \geq t}\left|k_{j}(x)\right| d \mu(x) & \leq\left\|\left(2^{j} x\right)^{k} k_{j}\right\|_{2}\left(\int_{x \geq t}\left(2^{j} x\right)^{-2 k} d \mu(x)\right)^{1 / 2} \\
& \leq C B\left(2^{j} t\right)^{(r+1) / 2-k} .
\end{aligned}
$$

If $\chi_{A}$ is the characteristic function of a set $A \subset \mathbf{R}^{+}$, then $0 \leq T^{y} \chi_{A}(x) \leq 1$ for $x, y \in \mathbf{R}^{+}$. We claim that

$$
T^{y_{0}} \chi_{\left\{z:\left|z-y_{0}\right|>2\left|y-y_{0}\right|\right\}} \leq \chi_{\left\{z: z>2\left|y-y_{0}\right|\right\}}
$$

and

$$
T^{y} \chi_{\left\{z: z-y_{0}|>2| y-y_{0} \mid\right\}} \leq \chi_{\left\{z ; z>\left|y-y_{0}\right|\right\}} \text {. }
$$

We only prove (3.9) and note that the proof of (3.10) is similar. Let $E$ denote the set $\left\{z:\left|z-y_{0}\right|>2\left|y-y_{0}\right|\right\}$. To verify (3.9) we need only check that $0 \leq x \leq 2\left|y-y_{0}\right|$ implies $T^{y_{0}} \chi_{E}(x)=0$. Since $0 \leq x \leq 2\left|y-y_{0}\right|$ implies $\left(\left|y_{0}-x\right|, y_{0}+x\right) \cap E=\varnothing$, it follows that $T^{y_{0}} \chi_{E}(x)=0$ (see remark after (2.6)). This proves (3.9).

Using (3.7) through (3.10) and the selfadjointness of generalized translations, cf. (2.3), we find that the left-hand side of (3.4) is majorized by

$$
\begin{aligned}
\int_{0}^{\infty} & \chi_{E}(x)\left|T^{y} k_{j}(x)\right| d \mu(x)+\int_{0}^{\infty} \chi_{E}(x)\left|T^{y_{0}} k_{j}(x)\right| d \mu(x) \\
& \leq \int_{\left|y-y_{0}\right|}^{\infty}\left|k_{j}(x)\right| d \mu(x)+\int_{2\left|y-y_{0}\right|}^{\infty}\left|k_{j}(x)\right| d \mu(x) \\
& \leq C B\left(2^{j}\left|y-y_{0}\right|\right)^{(r+1) / 2-k} .
\end{aligned}
$$

Thus (3.4) is established.

Finally, to establish (3.5) we use Bernstein's inequality. Since $\hat{k}_{j}=m_{j}$ has support in $\left(0,2^{j+1}\right)$ it follows from Corollary (2.2) and (3.7) that

$$
\begin{gathered}
\int_{\left|x-y_{0}\right| \geq 2\left|y-y_{0}\right|}\left|T^{y} k_{j}-T^{y_{0}} k_{j}\right| d \mu \leq\left\|T^{y} k_{j}-T^{y_{0}} k_{j}\right\|_{1} \\
\leq 2^{j+1}\left|y-y_{0}\right|\left\|k_{j}\right\|_{1} \leq C B 2^{j}\left|y-y_{0}\right| .
\end{gathered}
$$

Thus (3.5) is established. Combining (3.4) and (3.5) we obtain (3.3) which completes the proof of the theorem.

\section{A FINAL REMARK}

In connection with the Bernstein-type inequality (2.7) the following question arises. Consider the "mean value" operators $T^{y}, y \geq 0$, defined for functions on $\mathbf{R}^{n}, n \geq 2$, by

$$
T^{y} f(\bar{x})=\int_{S^{n-1}} f(\bar{x}+y \bar{t}) d \sigma(\bar{t})
$$


where $d \sigma$ is normalized surface measure on the sphere $S^{n-1}$. In other words $T^{y}$ is the convolution operator with the uniformly distributed probability measure on $\left\{\bar{x} \in \mathbf{R}^{n}:\|\bar{x}\|=y\right\}$. Is it true that for any $h \in C^{1}\left(\mathbf{R}^{n}\right)$ with, say, $\|\nabla h\| \in L^{1}\left(\mathbf{R}^{n}\right)$, the following estimate holds

$$
\left\|T^{y_{1}} h-T^{y_{2}} h\right\|_{L^{1}\left(\mathbf{R}^{n}\right)} \leq C\left|y_{1}-y_{2}\right|
$$

where $C$ depends on $h$ but not on $y_{1}$ and $y_{2}$ ? We note that Theorem 2.1 gives a positive answer to this problem only in the case when $h$ is radial.

But one can rather easily remark that the above problem may be answered affirmatively by writing out $\left\|T^{y_{1}} h-T^{y_{2}} h\right\|_{L^{1}\left(\mathbf{R}^{n}\right)}$, interchanging the order of integration and then applying the ordinary Bernstein-type inequality on $\mathbf{R}^{n}$. This yields an easy proof of Theorem 2.1 in the case of integral values of $r$.

\section{REFERENCES}

1. R. Coifman and G. Weiss, Analyse harmonique non commutative sur certains espaces homogènes, Lecture Notes in Math., vol. 242, Springer-Verlag, Berlin and New York, 1971.

2. R. E. Edwards and G. I. Gaudry, Littlewood-Paley and multiplier theory, Springer-Verlag, Berlin and New York, 1977.

3. L. Hörmander, Estimates for translation invariant operators in $L^{p}$ spaces, Acta Math. 104 (1960), 93-140.

4. K. Stempak, La théorie de Littlewood-Paley pour la transformation de Fourier-Bessel, C. R. Acad. Sci. Paris 303 (1986), 15-18.

5. no. $45,1985$.

Current address (John Gosselin): Department of Mathematics, University of Georgia, Athens, Georgia 30602

Current address (Krzysztof Stempak): InStitute of Mathematics, University of Wrociaw, Pl. Grunwaldzki 2/4, 50-384 Wroclaw, Poland 